\title{
The Value of Information Under Unawareness*
}

\author{
Spyros Galanis ${ }^{\dagger}$
}

January 23, 2015

\begin{abstract}
The value of information is examined in a single-agent environment with unawareness. Although the agent has a correct prior about events he is aware of and has a clear understanding of his available actions and payoffs, his unawareness may lead him to commit information processing errors and to behave suboptimally. As a result, the value of information can be negative, contrasting what is true in the standard model with partitional information and no unawareness. We show that the source of the agent's suboptimal behavior is that he misunderstands the information revealed by his varying awareness, treating it asymmetrically.
\end{abstract}

JEL-Classifications: C70, D01, D80, D82.

Keywords: unawareness, value of information, uncertainty, knowledge, bounded perception, awareness.

\section{Introduction}

Consider an agent who contemplates investing in the stock market today. His payoff is determined by the prices of shares tomorrow and his particular buy and sell orders. The agent is aware of all possible actions (investments) and prices of the shares.

Suppose now that there are other contingencies, expressed by questions, which indirectly influence the prices of shares tomorrow, and therefore the agent's payoff. Examples of such questions are whether there will be a merger, what are the characteristics of a new CEO or whether a specific innovation will be announced. The agent may be aware of some of these questions and unaware of others. Being unaware of a question means that he does not know its answer and he does not know that he does not know. In other words, he misses some information and at the same time fails to recognize it.

Although the agent has an incomplete understanding of the world and there are questions that he has never thought about, he receives correct information about events he is aware of and, within the bounds of his reasoning, he is perfectly rational and updates using Bayes' rule. Moreover, he is aware of all of his possible actions and he does not err when contemplating their deterministic payoff.

\footnotetext{
* Some of the results in this paper also appear in an earlier form in Galanis (2011b). I am grateful to two anonymous referees and the editor, Paulo Barelli, Piero Gottardi, Larry G. Epstein, Martin Meier, Herakles Polemarchakis, Marzena Rostek, David Rahman, Fernando Vega-Redondo, Marek Weretka, Xiaojian Zhao, seminar participants at the University of Bath, the European University Institute, the University of Southampton, the Summer in Birmingham workshop and the International Workshop on Unawareness, University of Queensland.

${ }^{\dagger}$ Department of Economics, School of Social Sciences, University of Southampton, Southampton, UK, s.galanis@soton.ac.uk.
} 
Is information valuable in such a setting? We study this question in an environment where an agent chooses an action, after receiving his awareness and information. We compare what is the effect of more informative signals on his ex ante expected utility.

As we show with an example in Section 2, the value of information can be negative. This is opposite to what is true when the agent is always fully aware and has a partitional information structure. In particular, Blackwell (1951) and Laffont (1989) show that more information is equivalent to having a higher ex ante expected utility from choosing an optimal action in the interim period, after receiving some information.

Since unawareness is a mistake of reasoning, this is not entirely surprising. Even within the standard framework, these phenomena can occur if agents have a non-partitional information structure, as in Geanakoplos (1989), or misinterpret their signals and have wrong posterior beliefs, as in Morris (1992) and Morris and Shin (1997). ${ }^{1}$ We cannot use the model of Geanakoplos (1989) to analyze the value of information under unawareness, because Dekel et al. (1998) have shown that a single state space can only express trivial unawareness. In order to overcome this criticism, we use the multiple state space model of unawareness developed in Galanis (2013), which is based on Heifetz et al. (2006).

The model in Morris (1992) and Morris and Shin (1997) is a reduced-form representation of beliefs. It allows all possible conditional beliefs, even wrong ones, without providing a theory of how they are formed. Because we need to derive conditional beliefs specifically generated by unawareness, we use a multiple state spaces model. We clarify this difference between our model and theirs at the end of Section 3, showing that (unlike them) unawareness does not generate all possible conditional beliefs, thus restricting behavior.

Moreover, Geanakoplos (1989) and Morris and Shin (1997) only compare an agent who makes some information processing errors with another who is less informed but is otherwise perfectly rational. In this paper we formulate results that allow for any agent, not just the more informed, to be unaware and to make information processing errors. Finally, Quiggin (2014) shows, in a model with unawareness where the value of information is always positive, that the sum of the gains in expected utility, going from no information to some information and from minimal awareness to full awareness, is always constant.

The main information processing error that we identify is that the agent, as he cannot reason beyond his awareness, treats his "awareness signal" asymmetrically. This signal summarizes the information revealed by the fact that his awareness varies across states. For example, when his awareness is high, he can exclude states describing that his awareness is low. However, when his awareness is low, he cannot exclude states describing that his awareness is high, because he cannot reason beyond his current level of awareness. Theorem 1 provides sufficient conditions that correct this problem, ensuring that the value of information is positive. Galanis (2014) shows that similar issues arise in risk-sharing environments, making the value of public information positive for some agents, when it is negative for all risk averse agents in the standard model.

As in almost all papers in the literature, the value of information is measured by the difference in the ex ante expected utility from increasing information. However, with unawareness there is a significant difference, as an unaware agent cannot realize that receiving more information ex ante could harm him. This is not a constraint of the model, but a constraint of unawareness. If the agent can understand that more information is bad, he is also able to single out the actions which are suboptimal in the interim period, which means that he has a perfect understanding of the relevant contingencies. But then, he is aware of these contingencies in the ex ante period

\footnotetext{
${ }^{1}$ In the standard framework that we refer to and in this paper, information is valuable if it allows the agent to choose better actions. However, there is also the possibility of having an intrinsic (i.e. for its own sake) value of information, that does not depend on one's actions. Grant et al. (1998) examine this case and provide several examples, some with negative value of information. For instance, expectant parents may be willing to pay not to learn the sex of their future child, even if their actions will not change whether they have this information or not.
} 
and does not make these suboptimal choices in the first place.

This implies that models of unawareness have nothing to say about how an agent can optimally choose between information structures in the ex ante period, as he does not expect that his posterior beliefs will change in the interim period, after becoming more aware. This is not the case for Morris (1992) and Morris and Shin (1997), because their agent understands ex ante what his posterior beliefs will be in the interim period after receiving a signal, even if they are not necessarily consistent with the true signal structure.

Although in our model the agent cannot understand ex ante the effect of more information on his welfare, he is aware of all of his available actions and the associated payoffs. Therefore, he can perfectly understand and evaluate ex ante any lottery over outcomes that is generated by an information structure and his actions in the interim period. What he cannot do is determine which lottery is associated with which information structure. In other words, there is nothing problematic about measuring the value of information using ex ante expected utility, even though the agent may have different levels of awareness in the interim period. The bounded rationality constraint imposed by unawareness is that he has an incomplete mapping between the available information structures and the lotteries over outcomes they generate.

The paper proceeds as follows. In Section 2 we show by example that information can be negatively valued in a single-agent environment. Section 3 presents the model and provides necessary and sufficient conditions for the value of information to be positive. The proofs are contained in the Appendix.

\section{An example}

We compare two agents who share the same awareness, payoffs, action set and prior, but one has more information and he is strictly worse off ex ante. Everything that is relevant about the world can be described by giving an answer to the following three questions. Question p, "What is the price of the share?", has three possible answers: low, medium and high. Questions $q$, "Is there going to be an acquisition?", and $r$, "Is there an innovation going to be adopted?", have two possible answers, "yes" and "no".

There are two available actions, buy (B) and not buy (NB). Payoffs depend only on the price. The payoff (in utils) is zero if the action is NB, irrespective of the price. If the action is $\mathrm{B}$ then the payoff is 1 if the price is high and -1 otherwise. Therefore, in terms of payoffs there is no difference between a low and a medium price.

A full state $s^{*}$ specifies an answer to all three questions and thus provides a complete description of the world. Let $S^{*}$ denote the full state space, the collection of full states. It contains the following three full states: $s_{1}^{*}=\left(p_{l}, q_{y}, r_{n}\right), s_{2}^{*}=\left(p_{m}, q_{n}, r_{y}\right)$, and $s_{3}^{*}=\left(p_{h}, q_{y}, r_{y}\right)$. Note that although payoffs depend only on prices, information about the other two questions can help an agent make a better decision. For example, if an agent knew that there is no acquisition $\left(q_{n}\right)$, he would understand that the price is medium $\left(p_{m}\right)$ and therefore he would choose NB.

Suppose now that both agents are always aware of questions $p$ and $q$, while they are aware of question $r$ if and only if state $s_{3}^{*}$ occurs. Being unaware of question $r$ means that you do not know whether there is an innovation, and you do not know that you do not know. In other words, the agent completely misses the $r$ dimension and his view of the world is represented by state space $S$, which consists of states $s_{1}=\left(p_{l}, q_{y}\right), s_{2}=\left(p_{m}, q_{n}\right)$ and $s_{3}=\left(p_{h}, q_{y}\right)$.

The two state spaces are depicted in Figure 1 . At full states $s_{1}^{*}$ and $s_{2}^{*}$, the downward arrows show that the agent is partially aware and his state space is $S$, whereas at $s_{3}^{*}$, the cyclical arrow shows that he is fully aware and his state space is $S^{*}$.

However, an unaware agent misses much more than just information about dimension $r$. The full state space $S^{*}$ specifies that the agent is aware of $r$ if and only if the price is high. This information is lost in state space $S$, because dimension $r$ is absent. In other words, at $s_{3}^{*}$, a fully aware agent can reason as follows: "I am aware of all three questions. Hence, the price is high". 


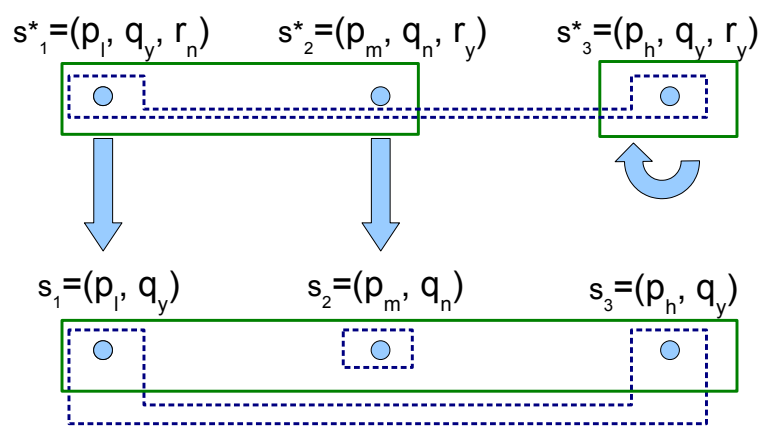

Figure 1: Awareness and information signals

But at $s_{1}^{*}$, an agent who is unaware of $r$ cannot reason as follows: "I am unaware of $r$. Hence, the price cannot be high".

In other words, if awareness is not constant across states, it creates an informative signal that the agent can only partially understand. We call this the awareness signal, depicted in Figure 1 by the partitions of $S^{*}$ and $S$, given by the solid lines. At $s_{1}^{*}$ and $s_{2}^{*}$, the agent's awareness is $S$, whereas at $s_{3}^{*}$, the agent is aware of $S^{*}$ and reasons that $s_{1}^{*}$ and $s_{2}^{*}$ cannot have happened, as they describe a lower awareness. Hence, the awareness signal, described in $S^{*}$, provides the informative partition $\left\{\left(s_{1}^{*}, s_{2}^{*}\right),\left(s_{3}^{*}\right)\right\}$. On the other hand, every state $s \in S$ describes that the agent's awareness is $S$, hence the awareness signal, described in $S$, provides the trivial partition $\left\{\left(s_{1}, s_{2}, s_{3}\right)\right\}$.

Although the two agents are identical in terms of their awareness, payoffs, prior and actions, they have different information. Suppose that agent 2 has a signal that always provides an answer to question $q$, making him more informed than agent 1 . This information signal is depicted in Figure 1 by the discontinuous lines.

At $s_{3}^{*}$, both agents use their awareness signal and deduce that the price is high. Agent 2's information signal is irrelevant here because it is less informative. At $s_{1}^{*}$, both agents are only aware of $S$. The less informed agent 1 cannot exclude any states because the awareness signal is completely uninformative for $S$, whereas agent 2 can use his information signal and exclude that the price is medium, as he knows that there is an acquisition. At $s_{2}^{*}$, again both agents are only aware of $S$ and the less informed agent 1 cannot exclude any states, whereas agent 2 can use his information signal to conclude that the price is medium, as he knows that there is no acquisition.

Let $\pi$ be the prior on the full state space $S^{*}$, such that $\pi\left(s_{1}^{*}\right)=\pi\left(s_{2}^{*}\right)=0.3$ and $\pi\left(s_{3}^{*}\right)=0.4$. The prior on $S$ is just the marginal of $\pi$. The following table summarizes, for each full state, the posteriors (and information) of each agent about the payoff relevant state space $\left(p_{l}, p_{m}, p_{h}\right)$ and their best action. For instance, at $s_{1}^{*}$ agent 2 assigns posterior probability of $3 / 7$ to the price being low, $4 / 7$ to the price being high and his best action is to buy.

\begin{tabular}{ccccc} 
Full state & Agent 1 & Action & Agent 2 & Action \\
\hline$s_{1}^{*}$ & $(0.3,0.3,0.4)$ & $\mathrm{NB}$ & $(3 / 7,0,4 / 7)$ & $\mathrm{B}$ \\
$s_{2}^{*}$ & $(0.3,0.3,0.4)$ & $\mathrm{NB}$ & $(0,1,0)$ & $\mathrm{NB}$ \\
$s_{3}^{*}$ & $(0,0,1)$ & $\mathrm{B}$ & $(0,0,1)$ & $\mathrm{B}$ \\
\hline
\end{tabular}

The less informed agent 1 always chooses the correct action, since he buys only when the price is high. On the contrary, the more informed agent 2 makes the wrong choice of buying when the price is low. Hence, he is strictly worse off ex ante. 
Note that both agents make the same information processing error, by treating their awareness signal asymmetrically. When the price is low, they do not exclude that the price is high, because they cannot reason beyond their awareness and realize that a high price implies high awareness. But when the price is high, they both exclude that the price is low, because a low price implies a low awareness.

We can ensure that the value of information is positive if we change the information structure in the following two ways. First, suppose that even if agent 2 was able to use his awareness signal at all states, his posterior beliefs would not change. Hence, his awareness signal cannot be treated asymmetrically, as it does not influence actions. We call this property Conditional Independence. It is satisfied if at $s_{1}^{*}$ he knows that $s_{1}$ has occurred and therefore chooses NB. His actions at $s_{2}^{*}$ and $s_{3}^{*}$ do not change and he is not ex ante worse off.

Second, suppose that agent 1 has a completely uninformative awareness signal. As a result, he always chooses NB. Agent 2 still treats his awareness signal asymmetrically, but his information signal is more informative than both the information and the awareness signals of 1 . Moreover, 2's awareness is path-independent, meaning in this example that his possible levels of awareness are ordered. Then, agent 2 is ex ante better off because he chooses B when the price is low or high, which is better than choosing NB at those states, as 1 does. We formalize these conditions and show in Theorem 1 that they are sufficient for information to be valuable.

\section{The Model}

\subsection{Preliminaries}

This section presents a reduced version of the model developed in Galanis (2013), which is based on the model of Heifetz et al. (2006). ${ }^{2}$ In particular, we only use possibility correspondences $P^{i}$ and not knowledge or awareness operators, as in the full-blown model.

Differences in awareness are modeled by having several state spaces. Let $\mathcal{S}=\left\{S_{a}\right\}_{a \in A}$ be a finite collection of disjoint state spaces, each having finitely many elements. We assume that $\mathcal{S}$ is a lattice with partial order $\preceq$, so that for every two elements $S, S^{\prime} \in \mathcal{S}$, both their supremum $S \vee S^{\prime}$ and their infimum $S \wedge S^{\prime}$ belong to $\mathcal{S}$. If $S \preceq S^{\prime}$, we say that $S^{\prime}$ is more expressive than $S$. Because $\mathcal{S}$ is finite, there is a top element, which we call the full state space $S^{*}$, and a bottom element, which we call the payoff relevant state space $S_{0}$. Hence, $S_{0} \preceq S \preceq S^{*}$, for all $S \in \mathcal{S}$. An event $E$ is a subset of some state space $S \in \mathcal{S}$.

If $S^{\prime \prime} \preceq S \preceq S^{\prime}$, we require that each state $s^{\prime} \in S^{\prime}$ can be mapped to its "restricted" image in the less expressive $S$, which we denote $s_{S}^{\prime} \in S$. We require that projections commute, so that $s_{S^{\prime \prime}}^{\prime}=\left(s_{S}^{\prime}\right)_{S^{\prime \prime}} \in S^{\prime \prime}$. Given an event $E \subseteq S$, let $E_{S^{\prime \prime}} \subseteq S^{\prime \prime}$ be its restriction to the less expressive $S^{\prime \prime}$, containing the restricted images in $S^{\prime \prime}$ of all states in $E$. Let $E^{S^{\prime}} \subseteq S^{\prime}$ be its enlargement to the more expressive $S^{\prime}$, containing all states in $S^{\prime}$ that, when restricted to $S$, belong to $E$. Let $E^{\uparrow}$ be the union of all enlargements $E^{S^{\prime}}$ to state spaces $S^{\prime}$ which are at least as expressive as $S$.

Agent $i$ 's information structure is represented by a possibility correspondence $P^{i}: \Sigma \rightarrow 2^{\Sigma} \backslash \emptyset$, where $\Sigma=\bigcup_{S \in \mathcal{S}} S$ is the set of all states. The interpretation is that $i$ considers $P^{i}(s)$ to be possible at $s \in S$. We assume that $P^{i}$ has the following properties.

(0) Confinedness: If $s \in S$ then $P^{i}(s) \subseteq S^{\prime}$ for some $S^{\prime} \preceq S$.

(1) Generalized Reflexivity: $s \in\left(P^{i}(s)\right)^{\uparrow}$ for every $s \in \Sigma$.

(2) Stationarity: $s^{\prime} \in P^{i}(s)$ implies $P^{i}\left(s^{\prime}\right)=P^{i}(s)$.

(3) Projections Preserve Ignorance: If $s \in S^{\prime}$ and $S \preceq S^{\prime}$ then $\left(P^{i}(s)\right)^{\uparrow} \subseteq\left(P^{i}\left(s_{S}\right)\right)^{\uparrow}$.

\footnotetext{
${ }^{2}$ For a comparison of the present model and other models of unawareness such as those of Heifetz et al. (2006) and Li (2009), see Galanis (2013) and its syntactic version Galanis (2011a).
} 
(4) Projections Preserve Awareness: If $s \in S^{\prime}, s \in P^{i}(s)$ and $S \preceq S^{\prime}$ then $s_{S} \in P^{i}\left(s_{S}\right)$.

Define $S^{i}: \Sigma \rightarrow \mathcal{S}$ such that for any $s \in \Sigma, S^{i}(s)=S$ if $P^{i}(s) \subseteq S \in \mathcal{S}$. $S^{i}(s)$ denotes agent $i$ 's state space at $s$. We say that $i$ is aware of event $E^{\prime} \subseteq S^{\prime}$ at $s$ if $S^{\prime} \preceq S^{i}(s)$.

\section{$3.2 \quad$ Priors and updating}

There are two periods, the ex ante period 0 and the interim period 1 . In period 0 , agent $i$ has some initial state space $S \in \mathcal{S}$, which describes how he views the uncertainty about period 1 . In period 1 , he receives some extra awareness and information, as specified by $P^{i}$.

Let $\pi_{0}$ be the prior on the full state space $S^{*}$. Because we want to attribute differences in posterior beliefs only on differences in awareness and information, the prior on any other state space $S$ is just the marginal of $\pi_{0}$. Formally, define $\pi: 2^{\Sigma} \backslash \emptyset \rightarrow[0,1]$ such that, for each event $E$, $\pi(E)=\pi_{0}\left(E^{S^{*}}\right)$. Posterior beliefs are formulated using Bayes' rule. If $i$ is aware of event $E$ at state $s$ and $\pi\left(P^{i}(s)\right)>0$, then his posterior belief about $E$ at $s$ is $\pi\left(E \mid P^{i}(s)\right)=\frac{\pi\left(E^{S^{i}(s)} \cap P^{i}(s)\right)}{\pi\left(P^{i}(s)\right)}$. We call $\pi$ a generalized prior.

\subsection{Information}

The extra complication that arises when comparing information structures $P^{1}$ and $P^{2}$ in an environment with unawareness is that $P^{1}(s)$ and $P^{2}(s)$ may belong to different state spaces. We compare them by enlarging them to a reference state space $S$.

Definition 1. $P^{2}$ is more informative than $P^{1}$ given $S \in \mathcal{S}$ if $P^{2}(s)^{S} \subseteq P^{1}(s)^{S}$ for all $s \in S .^{3}$

Note that each state space $S \in \mathcal{S}$ generates a different definition of what it means that $P^{2}$ is more informative than $P^{1}$. All subsequent definitions are similarly parametrized by a reference state space $S$.

\subsection{Awareness}

Three properties of awareness are relevant for the value of information. The first specifies that for any three states $s_{1}, s_{2}, s_{3} \in S$, if $s_{3}$ describes higher awareness than both $s_{1}$ and $s_{2}$, then the awareness described by $s_{1}$ is either higher or lower than the awareness described by $s_{2}$.

Definition 2. Awareness for $P^{i}$ is path-independent given $S \in \mathcal{S}$ if, for all $s_{1}, s_{2}, s_{3} \in S$, if $S^{i}\left(s_{3}\right) \succeq S^{i}\left(s_{1}\right)$ and $S^{i}\left(s_{3}\right) \succeq S^{i}\left(s_{2}\right)$ then either $S^{i}\left(s_{1}\right) \succeq S^{i}\left(s_{2}\right)$ or $S^{i}\left(s_{2}\right) \succeq S^{i}\left(s_{1}\right)$.

Path-independence says that there is a unique path that leads to each level of awareness. If $S^{i}(s)$ is the agent's state space (and awareness) at $s \in S$, there can be at most one (maximal) path of successively more expressive state spaces, $S^{i}\left(s_{1}\right) \prec S^{i}\left(s_{2}\right) \prec \ldots S^{i}\left(s_{n}\right) \prec S^{i}(s)$, where $s_{1}, \ldots, s_{n} \in S$, that lead to $S^{i}(s)$.

For the next two properties we first need to define what an unawareness signal is. Given state space $S \in \mathcal{S}$, for each $s \in S$, let $\mathcal{E}_{S}^{i}(s)=\left\{s_{1} \in S: S^{i}(s)=S^{i}\left(s_{1}\right)\right\}$ be the event in $S$ describing that the agent has the same awareness as in $s$. Operator $\mathcal{E}_{S}^{i}$ partitions state space $S$, generating an awareness signal, expressed in $S$, that provides information. We call $\mathcal{E}^{i}=\left\{\mathcal{E}_{S}^{i}\right\}_{S \in \mathcal{S}}$ the awareness signal of $P^{i}$.

The next property compares the information signal of $P^{i}$ with the awareness signal of $P^{j}$.

Definition 3. $P^{i}$ is more informative than the awareness signal of $P^{j}$ given $S \in \mathcal{S}$ if $P^{i}(s)^{S} \subseteq$ $\mathcal{E}_{S}^{j}(s)$ for all $s \in S$.

The final property, Conditional Independence, specifies that posterior beliefs, conditional on the information signal $P^{i}$, do not change when we also condition on the awareness signal of $P^{i}$.

\footnotetext{
${ }^{3}$ To ease the notation we write $P^{1}(s)^{S}$ instead of $\left(P^{1}(s)\right)^{S}$.
} 
Definition 4. $\left(P^{i}, \pi\right)$ satisfies Conditional Independence given $S \in \mathcal{S}$ if, for any $s \in S$ with $\pi(s)>0$, for any $E \subseteq S^{i}(s), \pi\left(E \mid P^{i}(s)\right)=\pi\left(E \mid \mathcal{E}_{S}^{i}(s) \cap P^{i}(s)^{S}\right)$.

\subsection{Single-agent decision problem}

Let $C$ be a finite set of possible actions and define $u: C \times \Sigma \rightarrow \mathbb{R}$ to be the agent's utility function. When the agent is aware of state $s$, his perception of his payoff at $s$ if he chooses action $c \in C$ is $u(c, s)$, which means that he is aware of all of the available actions in $C$. The following assumption requires that the agent has a correct understanding of the deterministic consequences of his actions. This is achieved by specifying that his deterministic payoffs are measurable with respect to the bottom state space, $S_{0}$, which he is always aware of.

Assumption 1. For all $s \in \Sigma$ and all $c \in C, u(c, s)=u\left(c, s_{S_{0}}\right)$.

The assumption has two implications. First, signals are not payoff relevant, as in Morris (1994): if two states $s, s^{\prime}$ project to the same state $s^{\prime \prime} \in S_{0}$, then they assign the same payoff for every action. Second, because all the payoff relevant events reside in $S_{0}$, the agent's priors about payoff relevant events are given by the marginal of $\pi$ on $S_{0}$.

These two properties are consistent with the story, outlined in the example, of an agent who invests in the stock market, he is aware of all of the available buy and sell orders and the payoff relevant state space consists of the prices of the stocks. The agent could form his priors about the payoff relevant events by looking at historical prices or by getting information about events he is already aware of, even if he is unaware of dimensions of the mechanism that generates the prices. Once he becomes aware of new dimensions and the associated events, he can go back and acquire new data about them, thus updating his posteriors about the payoff relevant events accordingly.

However, some interesting environments are excluded by Assumption 1 and therefore cannot be captured by the current model. Consider an agent who contemplates buying insurance for his house. ${ }^{4}$ He is aware of fire as a possible cause of destruction but unaware of earthquakes. Hence, he has an incorrect understanding of the deterministic consequences of his actions, because he thinks that if he does not buy insurance and there is no fire, his wealth will be intact. This is incorrect, so Assumption 1 is violated. In particular, in period 0 the agent is only aware of state space $S_{0}$, which contains two states, specifying whether there is a fire or not. But not all payoff relevant events reside in $S_{0}$. Because he mistakenly thinks that the payoff relevant event $E$, "my house is destroyed", is represented by the state describing that there is a fire, his prior underestimates $E$, as it excludes the state where an earthquake but not a fire has occurred.

A decision problem is a tuple $\left(S, P^{i}, C, u, \pi\right)$, where $S$ is a state space which describes the relevant uncertainty, $P^{i}$ is agent $i$ 's possibility correspondence, $C$ is the set of actions, $u$ is his utility function and $\pi$ is the generalized prior.

For every state space $S \in \mathcal{S}$ we define a collection of decision functions, $\mathcal{F}_{S}$, where each element is of the form $f: S \rightarrow C$. A decision function $f \in \mathcal{F}_{S}$ maps each state $s \in S$ to a specific action $c \in C$. We say that a decision function $f \in \mathcal{F}_{S}$ is measurable with respect to $P^{i}$ if, for all $s_{1}, s_{2} \in S, P^{i}\left(s_{1}\right)=P^{i}\left(s_{2}\right)$ implies $f\left(s_{1}\right)=f\left(s_{2}\right)$. It is optimal if it is measurable and prescribes the best action at each state $s \in S$.

Definition 5. Decision function $f \in \mathcal{F}_{S}$ is optimal for decision problem $\left(S, P^{i}, C, u, \pi\right)$ if it is measurable with respect to $P^{i}$ and, for all $s \in S$ and $c \in C$,

$$
\sum_{s^{\prime} \in P^{i}(s)} u\left(f(s), s^{\prime}\right) \pi\left(s^{\prime}\right) \geq \sum_{s^{\prime} \in P^{i}(s)} u\left(c, s^{\prime}\right) \pi\left(s^{\prime}\right) .
$$

${ }^{4}$ I thank a referee for pointing out this interesting example and its relation to Assumption 1. 
We compare two decision problems which differ only in terms of their information structures by calculating which guarantees a higher ex ante expected utility.

Definition 6. Decision problem $A=\left(S, P^{1}, C, u, \pi\right)$ is more valuable than decision problem $B=\left(S, P^{2}, C, u, \pi\right)$ if, whenever $f \in \mathcal{F}_{S}$ is optimal for $A$ and $g \in \mathcal{F}_{S}$ is optimal for $B$ we have

$$
\sum_{s \in S} u(f(s), s) \pi(s) \geq \sum_{s \in S} u(g(s), s) \pi(s) .
$$

\subsection{Conditions for information to be valuable}

In the standard framework with partitional information structures, $P^{2}$ is finer than $P^{1}$ if and only if it is more valuable for all $\pi, u$ and $C$ (Laffont (1989)). With unawareness this is no longer true, as we showed in Section 2. The following theorem summarizes the sufficient conditions for information to be valuable in an environment with unawareness.

Theorem 1. Suppose that $P^{2}$ is more informative than $P^{1}$, given $S \in \mathcal{S}$. Decision problem $\left(S, P^{2}, C, u, \pi\right)$ is more valuable than $\left(S, P^{1}, C, u, \pi\right)$ if one of the following is true:

1. $\left(P^{2}, \pi\right)$ satisfies Conditional Independence, given $S$, or,

2. Awareness for $P^{2}$ is path-independent and $P^{2}$ is more informative than the awareness signal of $P^{1}$, given $S$.

For the first part of the theorem, if the more informed agent 2 satisfies Conditional Independence, then he cannot treat his awareness signal asymmetrically, because his posterior beliefs (and as a result his actions) do not change when conditioning also on the awareness signal. In other words, his actions are also optimal for the partition on $S$ which is generated by the conjunction of his awareness and information signals. What remains to show is that the actions of the less informed agent 1 are measurable (but not necessarily optimal) with respect to the partition generated by his own information and awareness signals and that this partition is coarser than that generated for agent 2 . One can then invoke the standard arguments involving two partitions, one finer than the other, to show that the value of information is positive.

For the second part of the theorem, agent 2 may still treat his awareness signal asymmetrically, but only mildly. For instance, he could consider state $s_{3}$ to be possible at $s_{1}$, because he is unaware of question $r$, and consider $s_{1}$ to be impossible at $s_{3}$, because he is now aware of $r$. But path-independence excludes the "double mistake" of considering state $s_{2}$ but not $s_{3}$ to be possible at $s_{1}$ (because he is aware of $q$ but not $r$ ), while considering states $s_{2}$ but not $s_{1}$ to be possible at $s_{3}$ (because he is aware of $r$ but not $q$ ). To compensate for this mild error, we require that 2 is more informed not only in terms of 1 's information signal, but also in terms of 1's awareness signal. Then, even if agent 1 treats his own awareness signal asymmetrically, he cannot, by accident, choose better actions on average.

Conversely, we show that if $P^{2}$ always generates more valuable problems than $P^{1}$, then $P^{2}$ is more informative than $P^{1}$ when projected to the payoff relevant state space, $S_{0}$.

Proposition 1. Suppose that, for all $u, \pi$ and $C$, decision problem $\left(S, P^{2}, C, u, \pi\right)$ is more valuable than $\left(S, P^{1}, C, u, \pi\right)$. Then, $P^{2}(s)_{S_{0}} \subseteq P^{1}(s)_{S_{0}}$ for all $s \in S$.

Finally, we clarify how our model compares to that of Morris (1992) and Morris and Shin (1997). First, they also provide necessary and sufficient conditions for information to be valuable, specifying that the agent's posterior beliefs should not wander "too far" from the true distribution over state-message pairs.

Second, although in our model the agent can have "wrong" posterior beliefs due to unawareness, it is not the case (unlike in these two papers) that any posterior belief is allowed, meaning that unawareness imposes some restrictions on behavior. In particular, Bayesian updating and 
Assumption 1 imply the following weak form of Dynamic Consistency. Take two decision functions $f, g \in \mathcal{F}_{S_{0}}$ that prescribe the same action for all states not in the payoff relevant event $E \subseteq S_{0}$. Suppose that in period 1 the agent receives some awareness, so that his state space is $S_{1}$, and he only receives payoff relevant information $E \subseteq S_{0}$, so that $P(s)=E^{S_{1}}$. Then, the agent weakly prefers $f$ over $g$ in period 0 if and only if he weakly prefers $f$ over $g$ in period 1 .

This property is weaker than the standard version of Dynamic Consistency (e.g. Ghirardato (2002)), because it only applies to payoff relevant events and decision functions defined on $S_{0}$. It implies that if we could observe that the agent strictly preferred $f$ over $g$ in period 0 , then receiving purely payoff relevant information would not make him reverse his choice in period 1. This is not true in Morris (1992) and Morris and Shin (1997), because all wrong posterior beliefs are allowed and therefore the agent could reverse his choice.

\section{A Appendix}

Lemma 1. For any state space $S \in \mathcal{S}$, for any possibility correspondence $P^{i}$ and the resulting awareness signal $\mathcal{E}_{S}^{i},\left\{P^{i}(s)^{S} \cap \mathcal{E}_{S}^{i}(s)\right\}_{s \in S}$ is a partition of $S$. Moreover, if $P^{2}$ is more informative than $P^{1}$ given $S \in \mathcal{S}$, then partition $\left\{P^{2}(s)^{S} \cap \mathcal{E}_{S}^{2}(s)\right\}_{s \in S}$ is finer than $\left\{P^{1}(s)^{S} \cap \mathcal{E}_{S}^{1}(s)\right\}_{s \in S}$.

Proof. From Generalized Reflexivity, $s \in P^{i}(s)^{S} \cap \mathcal{E}_{S}^{i}(s)$. Suppose $s_{1} \in P^{i}(s)^{S} \cap \mathcal{E}_{S}^{i}(s)$. Then, $S^{i}(s)=S^{i}\left(s_{1}\right)$ and $\left\{s_{1}\right\}_{S^{i}(s)} \in P^{i}(s)$. Generalized Reflexivity implies $\left\{s_{1}\right\}_{S^{i}(s)} \in P^{i}\left(s_{1}\right)$ and Stationarity implies $P^{i}\left(s_{1}\right)=P^{i}\left(\left\{s_{1}\right\}_{S^{i}(s)}\right)=P^{i}(s)$. Hence, $P^{i}\left(s_{1}\right)^{S} \cap \mathcal{E}_{S}^{i}\left(s_{1}\right)=P^{i}(s)^{S} \cap \mathcal{E}_{S}^{i}(s)$.

For the second claim, suppose $s_{1} \in P^{2}(s)^{S} \cap \mathcal{E}_{S}^{2}(s)$. Because $P^{2}$ is more informative than $P^{1}$ given $S$, we have $s_{1} \in P^{1}(s)^{S}$. Stationarity and Projections Preserve Ignorance imply that $S^{1}\left(s_{1}\right) \succeq S^{1}(s)$. Suppose $s_{1} \notin \mathcal{E}_{S}^{1}(s)$. Then, $S^{1}\left(s_{1}\right) \succ S^{1}(s)$, which implies that $s \notin P^{1}\left(s_{1}\right)^{S}$. Because $\left\{P^{2}(s)^{S} \cap \mathcal{E}_{S}^{2}(s)\right\}_{s \in S}$ is a partition, we have $P^{2}(s)^{S} \cap \mathcal{E}_{S}^{2}(s)=P^{2}\left(s_{1}\right)^{S} \cap \mathcal{E}_{S}^{2}\left(s_{1}\right)$, so together with Generalized Reflexivity we have $s \in P^{2}\left(s_{1}\right)^{S} \subseteq P^{1}\left(s_{1}\right)^{S}$, a contradiction.

Proof of the first part of Theorem 1. Suppose $f$ is optimal for $\left(S, P^{1}, C, u, \pi\right)$ and $g$ is optimal for $\left(S, P^{2}, C, u, \pi\right)$. Fix $s \in S$ with $\pi(s)>0$. For all $c \in C$, we have $\sum_{s_{1} \in P^{2}(s)} u\left(g(s), s_{1}\right) \pi\left(s_{1}\right) \geq$ $\sum_{s_{1} \in P^{2}\left(s_{1}\right)} u\left(c, s_{1}\right) \pi\left(s_{1}\right)$. Conditional Independence and Generalized Reflexivity imply that, for

$s_{1}, s_{2} \in P^{2}(s)$ such that $\pi\left(s_{1}\right), \pi\left(s_{2}\right)>0$, we have $\frac{\pi\left(\mathcal{E}_{S}^{2}(s) \cap s_{1}^{S}\right)}{\pi\left(s_{1}\right)}=\frac{\pi\left(\mathcal{E}_{S}^{2}(s) \cap s_{2}^{S}\right)}{\pi\left(s_{2}\right)}>0$. Multiplying by that number we have that, for all $c \in C$,

$$
\begin{aligned}
& \sum_{s_{1} \in P^{2}(s)} u\left(g(s), s_{1}\right) \pi\left(\mathcal{E}_{S}^{2}(s) \cap s_{1}^{S}\right) \geq \sum_{s_{1} \in P^{2}(s)} u\left(c, s_{1}\right) \pi\left(\mathcal{E}_{S}^{2}(s) \cap s_{1}^{S}\right) \Longrightarrow \\
& \sum_{s_{1} \in P^{2}(s)} u\left(g(s), s_{1}\right) \sum_{s_{2} \in \mathcal{E}_{S}^{2}(s) \cap s_{1}^{S}} \pi\left(s_{2}\right) \geq \sum_{s_{1} \in P^{2}(s)} u\left(c, s_{1}\right) \sum_{s_{2} \in \mathcal{E}_{S}^{2}(s) \cap s_{1}^{S}} \pi\left(s_{2}\right) .
\end{aligned}
$$

Since $\left\{s_{1}\right\}_{S_{0}}=\left\{s_{2}\right\}_{S_{0}}$ for all $s_{2} \in s_{1}^{S}$ we have

$$
\begin{gathered}
\sum_{s_{1} \in P^{2}(s)} \sum_{s_{2} \in \mathcal{E}_{S}^{2}(s) \cap s_{1}^{S}} u\left(g(s), s_{2}\right) \pi\left(s_{2}\right) \geq \sum_{s_{1} \in P^{2}(s)} \sum_{s_{2} \in \mathcal{E}_{S}^{2}(s) \cap s_{1}^{S}} u\left(c, s_{2}\right) \pi\left(s_{2}\right) \Longrightarrow \\
\sum_{s_{2} \in P^{2}(s)^{S} \cap \mathcal{E}_{S}^{2}(s)} u\left(g(s), s_{2}\right) \pi\left(s_{2}\right) \geq \sum_{s_{2} \in P^{2}(s)^{S} \cap \mathcal{E}_{S}^{2}(s)} u\left(c, s_{2}\right) \pi\left(s_{2}\right) .
\end{gathered}
$$


Hence, Conditional Independence implies that for any $s \in S$, the agent's best action at $P^{2}(s)$ is also the best action at $P^{2}(s)^{S} \cap \mathcal{E}_{S}^{2}(s)$. From Lemma $1,\left\{P^{2}(s)^{S} \cap \mathcal{E}_{S}^{2}(s)\right\}_{s \in S}$ is a partition of $S$ and it is finer than partition $\left\{P^{1}(s)^{S} \cap \mathcal{E}_{S}^{1}(s)\right\}_{s \in S}$.

We also show that $s_{1}, s_{2} \in P^{2}(s)^{S} \cap \mathcal{E}_{S}^{2}(s)$ implies $g\left(s_{1}\right)=g\left(s_{2}\right)$. Since $S^{2}\left(s_{1}\right)=S^{2}\left(s_{2}\right)=$ $S^{2}(s)$, Stationarity implies that either $P^{2}\left(s_{1}\right)=P^{2}(s)$ or $P^{2}\left(s_{1}\right) \cap P^{2}(s)=\emptyset$. Generalized Reflexivity and $s_{1} \in P^{2}(s)^{S}$ imply $P^{2}\left(s_{1}\right)=P^{2}(s)$. Similarly for $s_{2}$, so we have $P^{2}\left(s_{1}\right)=$ $P^{2}\left(s_{2}\right)$. Hence, $g\left(s_{1}\right)=g\left(s_{2}\right)$. The same argument shows that $s_{1}, s_{2} \in P^{1}(s)^{S} \cap \mathcal{E}_{S}^{1}(s)$ implies $f\left(s_{1}\right)=f\left(s_{2}\right)$.

We showed that for each element of the partition $\left\{P^{2}(s)^{S} \cap \mathcal{E}_{S}^{2}(s)\right\}_{s \in S}$, agent 2 picks an action that maximizes his expected utility. Moreover, agent 1 picks one action for each element of the partition, $\left\{P^{1}(s)^{S} \cap \mathcal{E}_{S}^{1}(s)\right\}_{s \in S}$. This action may not necessarily be optimal for that partition cell, as Conditional Independence is not satisfied for $\left(P^{1}, \pi\right)$.

Decision function $g$ is optimal for partition $\left\{P^{2}(s)^{S} \cap \mathcal{E}_{S}^{2}(s)\right\}_{s \in S}$, whereas $\mathrm{f}$ is measurable (but not necessarily optimal) for partition $\left\{P^{1}(s)^{S} \cap \mathcal{E}_{S}^{1}(s)\right\}_{s \in S}$. Moreover, the former partition is finer than the latter. Hence, the ex ante expected utility for 2 is higher and decision problem $\left(S, P^{2}, C, u, \pi\right)$ is more valuable than $\left(S, P^{1}, C, u, \pi\right)$.

Before proving the second part of Theorem 1, define the mapping $P: S \rightarrow 2^{S}$ such that for all $s \in S, P(s)=P^{2}(s)^{S}$. Consider the following three properties, discussed in Geanakoplos (1989), where $s, s_{1}, s_{2} \in S$. Non-delusion requires that $s \in P(s)$. KTYK requires that if $s_{1} \in P(s)$, then $P\left(s_{1}\right) \subseteq P(s)$. Nested requires that either $P\left(s_{1}\right) \cap P\left(s_{2}\right)=\emptyset$, or $P\left(s_{1}\right) \subseteq P\left(s_{2}\right)$ or $P\left(s_{2}\right) \subseteq P\left(s_{1}\right)$.

Lemma 2. $P$ satisfies non-delusion and KTYK. If awareness for $P^{2}$ is path-independent, then $P$ satisfies nested.

Proof. $P$ satisfies non-delusion because $P^{2}$ satisfies Generalized Reflexivity. For KTYK, suppose $s_{1} \in P(s)=P^{2}(s)^{S}$. Then, $\left\{s_{1}\right\}_{S^{2}(s)} \in P^{2}(s)$. Stationarity implies $P^{2}\left(\left\{s_{1}\right\}_{S^{2}(s)}\right)=P^{2}(s)$. Projections Preserve Ignorance implies $P^{2}\left(s_{1}\right)^{S} \subseteq\left(P^{2}\left(\left\{s_{1}\right\}_{S^{2}(s)}\right)\right)^{S}=P^{2}(s)^{S}$. Hence $P\left(s_{1}\right) \subseteq$ $P(s)$. For nested, suppose $P\left(s_{1}\right) \cap P\left(s_{2}\right) \neq \emptyset$. Take $s_{3} \in P\left(s_{1}\right) \cap P\left(s_{2}\right)$. Then $s_{3} \in P^{2}\left(s_{1}\right)^{S} \cap$ $P^{2}\left(s_{2}\right)^{S}$. As in the previous paragraph, Generalized Reflexivity and Stationarity imply that $P^{2}\left(\left\{s_{3}\right\}_{S^{2}\left(s_{2}\right)}\right)=P^{2}\left(s_{2}\right)$ and $P^{2}\left(\left\{s_{3}\right\}_{S^{2}\left(s_{1}\right)}\right)=P^{2}\left(s_{1}\right)$. Projections Preserve Ignorance implies that $S^{2}\left(s_{3}\right) \succeq S^{2}\left(s_{1}\right)$ and $S^{2}\left(s_{3}\right) \succeq S^{2}\left(s_{2}\right)$. Since awareness for $P^{2}$ is path-independent, suppose, without loss of generality, that $S^{2}\left(s_{1}\right) \preceq S^{2}\left(s_{2}\right)$. Projections Preserve Ignorance implies that $P^{2}\left(\left\{s_{3}\right\}_{S^{2}\left(s_{2}\right)}\right)^{S} \subseteq P^{2}\left(\left\{s_{3}\right\}_{S^{2}\left(s_{1}\right)}\right)^{S}$, hence $P\left(s_{2}\right) \subseteq P\left(s_{1}\right)$.

Proof of the second part of Theorem 1. Suppose $f$ is optimal for $\left(S, P^{1}, C, u, \pi\right)$ and $g$ is optimal for $\left(S, P^{2}, C, u, \pi\right)$. Define $Q: S \rightarrow 2^{S}$ such that $Q(s)=P^{1}(s)^{S} \cap \mathcal{E}_{S}^{1}(s)$. From Lemma 1 and the proof of the first part of Theorem $1, Q$ is a partition of $S$ and $f$ is measurable with respect to $Q$. Define $P: S \rightarrow 2^{S}$ such that for all $s \in S, P(s)=P^{2}(s)^{S}$. By Lemma $2, P$ satisfies non-delusion, KTYK and nested. Since $P^{2}$ is more informative than $P^{1}$ and the awareness signal of $P^{1}$, given $S$, we have that $P$ is finer than $Q$. Moreover, $g$ is optimal for $(S, P, C, u, \pi)$ and $f$ is measurable (but not necessarily optimal) for $(S, Q, C, u, \pi)$.

In order to show that $\left(C, S, P^{2}, u, \pi\right)$ is more valuable than $\left(S, P^{1}, C, u, \pi\right)$, we adapt the proof of Theorem 1 in Geanakoplos (1989), to accommodate the fact that $f$ is measurable but not optimal, as it is assumed in Geanakoplos (1989). We proceed by induction on the cardinality of the finite state space $S$.

If $S$ consists of only one state, the claim is true. Assume that when $S$ consists of $k>1$ elements, the claim is true and therefore we have $\sum_{s \in S} u(g(s), s) \pi(s) \geq \sum_{s \in S} u(f(s), s) \pi(s)$.

Suppose that $S$ consists of $k+1$ elements. Let $E_{1}=\{s \in S: P(s)=S\}$. If $E_{1}=S$, then $Q$ is also the trivial partition because it is coarser. Since both $f$ and $g$ are measurable 
we are done. If $E_{1} \neq S$, define $E_{2}=P(s)$, where $P(s)$ has the largest number of states less than $k+1$. Let $E_{3}=S \backslash\left(E_{1} \cup E_{2}\right)$. Applying arguments similar to the proof of Theorem 1 in Geanakoplos (1989), we can show that since $P$ satisfies non-delusion, KTYK and nested, $E_{1}, E_{2}, E_{3}$ are disjoint and their union is $S$. Moreover, if $s \in E_{i}$ then $P(s) \subseteq E_{i}$, for $i=2,3$. This means that we can view $E_{2}$ and $E_{3}$ as separate state spaces, with cardinality less than $k+1$, where the restriction of $P$, denoted $P_{E_{2}}$, satisfies non-delusion, KTYK and nested, and similarly for $E_{3}$. Because $Q$ partitions $S$, the restriction of $Q$, denoted $Q_{E_{2}}$, partitions $E_{2}$, and similarly for $E_{3}$. Hence, $f$ is measurable in the restricted problem $\left(E_{1}, Q_{E_{i}}, C, u, \pi\right)$ and $g$ is optimal in $\left(E_{1}, P_{E_{i}}, C, u, \pi\right)$, for $i=1,2,3$. By applying the induction hypothesis, we have

$$
\begin{aligned}
& \sum_{s \in E_{1}} u(g(s), s) \pi(s)+\sum_{s \in E_{2}} u(g(s), s) \pi(s)+\sum_{s \in E_{3}} u(g(s), s) \pi(s) \geq \\
& \sum_{s \in E_{1}} u(f(s), s) \pi(s)+\sum_{s \in E_{2}} u(f(s), s) \pi(s)+\sum_{s \in E_{3}} u(f(s), s) \pi(s) .
\end{aligned}
$$

Because $S$ is partitioned by $E_{1}, E_{2}$ and $E_{3}$, we are done.

Proof of Proposition 1. To prove by contradiction, suppose that there exist $s \in S, s_{1} \in S_{0}$, such that $s_{1} \in P^{2}(s)_{S_{0}}$ and $s_{1} \notin P^{1}(s)_{S_{0}}$. By Generalized Reflexivity, $s_{S_{0}} \in P^{1}(s)_{S_{0}}$, so $s_{S_{0}} \neq s_{1}$. Let $s_{2} \in P^{2}(s)$ such that $s_{2 S_{0}}=s_{1}$. Let $s_{3} \in S$ such that $s_{3 S(s)}=s_{2}$. Define $\pi$ such that $\pi(s)=\pi\left(s_{3}\right)=1 / 2$. Hence, $\pi\left(s_{1}\right)=\pi\left(s_{2}\right)=1 / 2$. Let the action set be $C=\left\{c_{1}, c_{2}\right\}$ and consider the following payoffs: $u\left(c_{1}, s_{S_{0}}\right)=-1, u\left(c_{1}, s_{2 S_{0}}\right)=1.1, u\left(c_{2}, s_{S_{0}}\right)=8, u\left(c_{2}, s_{2 S_{0}}\right)=-8$. At state $s$, agent 2 considers both $s_{S_{0}}$ and $s_{2 S_{0}}$ to be possible and his optimal action is $c_{1}$. Agent 1 considers only $s_{S_{0}}$ to be possible and his optimal action is $c_{2}$. At $s_{3}$, from Generalized Reflexivity, both agents assign probability at least $1 / 2$ to state $s_{3 S_{0}}=s_{2 S_{0}}$ and their optimal action is $c_{1}$. Hence, the decision problem $\left(S, P^{1}, C, u, \pi\right)$ is more valuable than $\left(S, P^{2}, C, u, \pi\right)$, a contradiction.

\section{References}

David Blackwell. Comparison of experiments. In Proceedings of the Second Berkeley Symposium on Mathematical Statistics and Probability, pages 93-102. University of California Press, 1951.

Eddie Dekel, Bart Lipman, and Aldo Rustichini. Standard state spaces preclude unawareness. Econometrica, 66:159-173, 1998.

Spyros Galanis. Syntactic foundations for unawareness of theorems. Theory and Decision, 71 (4):593-614, 2011a.

Spyros Galanis. Trade and the value of information under unawareness. University of Southampton, Discussion Papers in Economics and Econometrics, 1313, 2011b.

Spyros Galanis. Unawareness of theorems. Economic Theory, 52(1):41-73, 2013.

Spyros Galanis. The value of information in risk-sharing environments with unawareness. Mimeo, 2014.

John Geanakoplos. Game theory without partitions, and applications to speculation and consensus. Cowles Foundation Discussion Paper, No. 914, 1989. 
Paolo Ghirardato. Revisiting Savage in a conditional world. Economic Theory, 20(1):83-92, 2002.

Simon Grant, Atsushi Kajii, and Ben Polak. Intrinsic Preference for Information. Journal of Economic Theory, 83(2):233-259, December 1998.

Aviad Heifetz, Martin Meier, and Burkhard C. Schipper. Interactive unawareness. Journal of Economic Theory, 130:78-94, 2006.

Jean-Jacques Laffont. The Economics of Uncertainty and Information. MIT Press, 1989.

Jing Li. Information structures with unawareness. Journal of Economic Theory, 144:977-993, 2009.

Stephen Morris. Revising beliefs and knowledge: A generalization of Blackwell's theorem. $C A$ RESS Working Paper \#92-24, 1992.

Stephen Morris. Trade with heterogeneous prior beliefs and asymmetric information. Econometrica, 62(6):1327-1347, November 1994.

Stephen Morris and Hyun Song Shin. The rationality and efficacy of decisions under uncertainty and the value of an experiment. Economic Theory, 9:309-324, 1997.

John Quiggin. The value of information and the value of awareness. Mimeo, 2014. 